\title{
Controlled delivery of PDGF-BB for myocardial protection using injectable self-assembling peptide nanofibers
}

\author{
Patrick C.H. Hsieh, Michael E. Davis, Joseph Gannon, Catherine MacGillivray, and Richard T. Lee \\ Cardiovascular Division, Department of Medicine, Brigham and Women's Hospital, Harvard Medical School, Boston, Massachusetts, USA.
}

\begin{abstract}
Endothelial cells can protect cardiomyocytes from injury, but the mechanism of this protection is incompletely described. Here we demonstrate that protection of cardiomyocytes by endothelial cells occurs through PDGF-BB signaling. PDGF-BB induced cardiomyocyte Akt phosphorylation in a time- and dose-dependent manner and prevented apoptosis via PI3K/Akt signaling. Using injectable self-assembling peptide nanofibers, which bound PDGF-BB in vitro, sustained delivery of PDGF-BB to the myocardium at the injected sites for 14 days was achieved. A blinded and randomized study in 96 rats showed that injecting nanofibers with PDGF-BB, but not nanofibers or PDGF-BB alone, decreased cardiomyocyte death and preserved systolic function after myocardial infarction. A separate blinded and randomized study in 52 rats showed that PDGF-BB delivered with nanofibers decreased infarct size after ischemia/reperfusion. PDGF-BB with nanofibers induced PDGFR- $\beta$ and Akt phosphorylation in cardiomyocytes in vivo. These data demonstrate that endothelial cells protect cardiomyocytes via PDGF-BB signaling and that this in vitro finding can be translated into an effective in vivo method of protecting myocardium after infarction. Furthermore, this study shows that injectable nanofibers allow precise and sustained delivery of proteins to the myocardium with potential therapeutic benefits.
\end{abstract}

\section{Introduction}

Recent studies have generated unprecedented excitement regarding potential advances in cardiac repair (1-4). Despite this enthusiasm, the mechanisms for potential beneficial effects of cardiac repair are largely unclear, underscoring the need for understanding the fundamental biology of myocardial protection and regeneration. The dominant cause of heart failure is regional loss of myocardium due to coronary artery disease and ischemia that leads to cardiomyocyte necrosis and apoptosis $(5,6)$. Therefore, therapies that protect cardiomyocytes from death may prevent the development of heart failure after ischemic injury.

In normal mammalian myocardium, cardiomyocytes are surrounded by an intricate network of capillaries. Endothelial cellcardiomyocyte interactions regulate not only cardiac development (7-11) but also cardiomyocyte function in the adult heart (12). Although the mechanisms by which endothelial cells may preserve cardiac function after ischemic injury are incompletely described, direct injection of bone marrow-derived angioblasts (13) or endothelial progenitor cells (14) after myocardial infarction (MI) may improve cardiac performance. In addition, in endothelial cell-cardiomyocyte coculture, endothelial cells may promote cardiomyocyte survival $(15,16)$. These results suggest that understanding myocardial protection by endothelial cells may lead to therapeutic applications to protect cardiomyocyte loss.

PDGF signaling plays an essential role in cardiovascular development (17). Deletion of the gene for PDGF-B or its principal receptor, PDGFR- $\beta$, leads to cardiac abnormalities that include late embryonic ventricular dilation, myocardial hypertrabeculation, and septal

Nonstandard abbreviations used: I/R, ischemia/reperfusion; MI, myocardial infarction; NF, nanofiber; P50, $50 \mathrm{ng} / \mathrm{ml}$ PDGF-BB.

Conflict of interest: The authors have declared that no conflict of interest exists.

Citation for this article: J. Clin. Invest. 116:237-248 (2006)

doi:10.1172/JCI25878. abnormalities $(18,19)$. Interestingly, endothelium-restricted ablation of PDGF-B results in the same myocardial anomalies as well as defects in vasculogenesis due to pericyte loss (20). It has also been shown that PDGF mediates cardiac microvascular endothelial cell communication with cardiomyocytes (21), and injection of PDGF may restore impaired endothelial PDGF-regulated angiogenesis and enhance postischemic neovascularization $(22,23)$. These data suggest that PDGF signaling could be a crucial mechanism of endothelial cell-cardiomyocyte communication.

The goal of the present study was to characterize cardiomyocyte survival promoted by endothelial cells and to design a controlleddelivery strategy for myocardial protection using self-assembling peptide nanofibers (NFs). Self-assembling peptide NFs are short peptides that assemble into stable NFs upon exposure to physiological $\mathrm{pH}$ and osmolarity; these properties allow the peptides to be injected for creation of tissue microenvironments consisting of NFs (24). Since peptide NFs can be modified in a variety of ways, this approach may allow delivery of factors to the myocardium in a highly controlled fashion. Here we demonstrate in rats that PDGF-BB/PDGFR- $\beta$ signaling functions as an endotheliumderived prosurvival pathway to prevent cardiomyocyte apoptosis through PI3K/Akt signaling. We then show that self-assembling NFs bind PDGF-BB, allowing prolonged delivery of PDGF-BB to the infarcted myocardium for up to 14 days. This strategy enabled protection of cardiomyocytes, reduced infarct size, and preserved systolic function after coronary ligation. Thus, delivery of PDGF$\mathrm{BB}$ using injectable self-assembling peptide NFs may provide a novel approach for myocardial protection.

\section{Results}

Endothelial cells promote cardiomyocyte survival via PDGF-BB/PDGFR- $\beta$ signaling. Previous studies demonstrated that endothelial cells can prevent cardiomyocyte apoptosis in coculture $(15,16)$, a finding we explored further to establish conditions for quantitative deliv- 
A

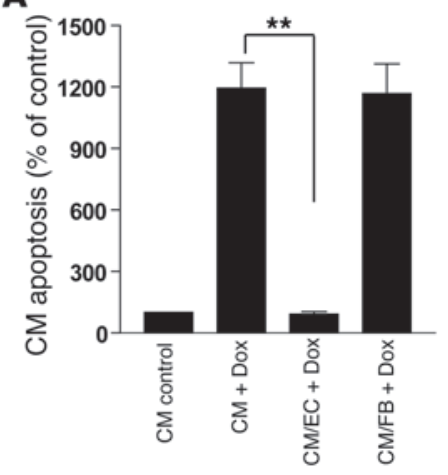

B

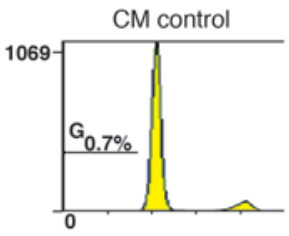

DNA fragmentation
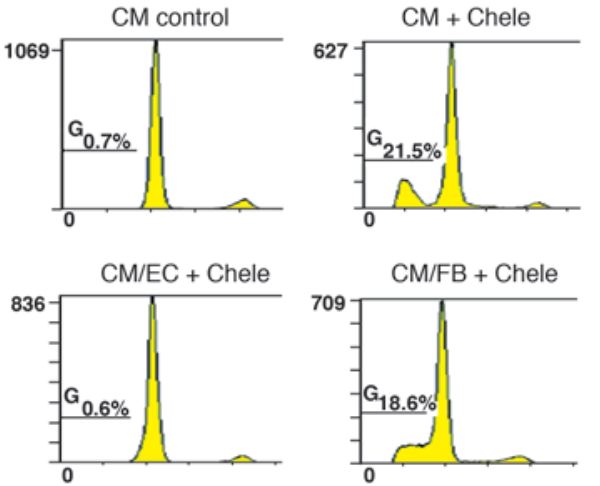

C
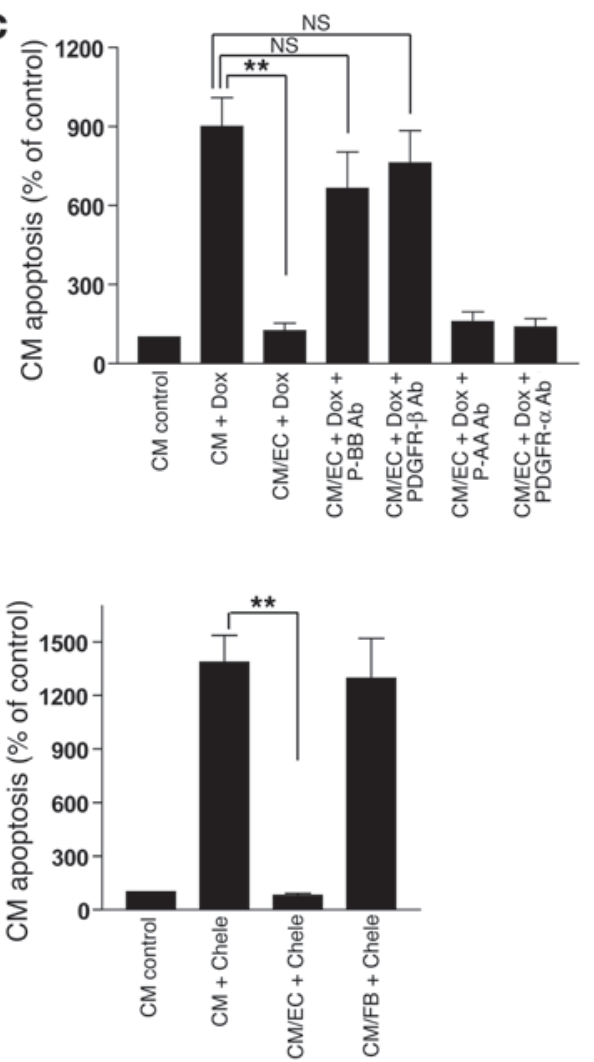

Figure 1

Endothelial cells promote cardiomyocyte survival via PDGF-BB signaling. Cardiomyocytes $(\mathrm{CM})$, cocultured with endothelial cells (EC) or fibroblasts (FB), were treated with doxorubicin (Dox) (A) or chelerythrine (Chele) (B) to induce apoptosis. TUNEL (A) and DNA fragmentation cytometry $(\mathbf{B})$ were used to quantify cardiomyocyte apoptosis with $\alpha$-sarcomeric actinin staining to identify cardiomyocytes. Arrows indicate areas of DNA fragmentation. $G$ indicates gated area for quantifying the DNA fragmentation. (C) Cardiomyocyte apoptosis, with or without endothelial coculture, was induced by doxorubicin with or without neutralizing antibodies against PDGF-BB (P-BB), PDGF-AA (P-AA), PDGFR- $\beta$, or PDGFR- $\alpha$, and was determined using propidium iodide cytometry. ${ }^{* *} P<0.001$. ery in vivo (Figure 1). Neonatal rat cardiomyocytes were cocultured with adult cardiac endothelial cells or fibroblasts, and cardiomyocyte apoptosis was induced by 3 different methods, including treatment with doxorubicin, chelerythrine, and $\mathrm{H}_{2} \mathrm{O}_{2}$ plus TNF- $\alpha$. Apoptosis was quantified with 3 independent assays, including in situ TUNEL staining, DNA fragmentation by propidium iodide staining, and annexin- $V$ cell sorting. With each apoptotic stimulus, coculture with endothelial cells, but not fibroblasts, prevented cardiomyocyte apoptosis (Figure 1, A and B, and data not shown). The protection of cardiomyocytes from apoptosis in cardiomyocyte-endothelial coculture was blocked by neutralizing antibodies against PDGF-BB or PDGFR- $\beta$, but not by antibodies against PDGF-AA or PDGFR- $\alpha$ (Figure 1C), indicating that endotheliumderived cardiomyocyte protection occurs through the PDGF-BB/ PDGFR- $\beta$ pathway.

PDGF-BB prevents cardiomyocyte apoptosis via PI3K/Akt signaling. To evaluate further the effects of PDGF-BB in cardiomyocyte apoptosis, we induced cardiomyocyte apoptosis by multiple methods with or without PDGF-BB and quantified apoptosis with 3 independent assays as described above. PDGF-BB prevented cardiomyocyte apoptosis irrespective of stimulus or assay, and there was a dosedependent antiapoptotic effect, with optimal results at PDGF-BB concentrations of $10 \mathrm{ng} / \mathrm{ml}$ and higher (doxorubicin results are shown in Figure 2A). PDGF-BB, but not PDGF-AA, induced phosphorylation of PDGFR- $\beta$ and the downstream kinase Akt in cardiomyocytes in a dose- and time-dependent manner (Figure 2B) but induced no significant activation of p42/p44 (ERK-1/2), p38, or jun $\mathrm{N}$-terminal kinase from 5 to 60 minutes (data not shown). Immunofluorescence costaining of phospho-PDGFR- $\beta$ and $\alpha$-sarcomeric actinin confirmed the activation and internal- ization of PDGFR- $\beta$ in differentiated cardiomyocytes by PDGF-BB (Figure 2C). Blocking Akt signaling using a PI3K-specific inhibitor (LY294002) or a dominant-negative Akt adenovirus abolished the prosurvival effect of PDGF-BB in cardiomyocytes (Figure 2D), demonstrating that cardiomyocyte protection of PDGF-BB may occur via the PI3K/Akt pathway. These results show that PDGF-BB is a potential candidate cardiomyocyte survival factor for targeted delivery into the myocardium.

Controlled intramyocardial delivery of PDGF-BB using injectable self-assembling peptide NFs. To explore the possibility of using selfassembling peptide NFs for controlled intramyocardial delivery of PDGF-BB, we first tested the binding capability of PDGF-BB by peptide NFs. Although these NFs do not have specific binding motifs for peptides, they are amphiphilic, with the potential to bind other proteins through weak molecular interactions. When PDGF-BB (100 ng total) was embedded with the NFs, the binding capacity was $1 \mathrm{ng}$ PDGF-BB per microgram of NFs (Figure $3 A)$. This binding capacity was 10 -fold higher than the amount of PDGF-BB per mass of NFs used in subsequent experiments in vivo (described below). We observed similar binding of other growth factors, including VEGF-A, bFGF, and angiopoietin-1, all of which bound significantly better to the peptide NFs than BSA.

To assess the feasibility of delivering PDGF-BB with self-assembling peptide NFs into the myocardium for cardioprotection, we injected PDGF-BB with peptide NFs into the myocardium of rats. All of the procedures were blinded and randomized, with at least 6 animals in each group. Left coronary arteries were ligated, immediately followed by direct myocardial injection of $1 \%$ peptide NFs (peptide sequence AcN-RARADADARARADADA-CNH $\mathrm{H}_{2}$ ) with or without PDGF-BB into the infarcted border zones at 3 loca- 
A

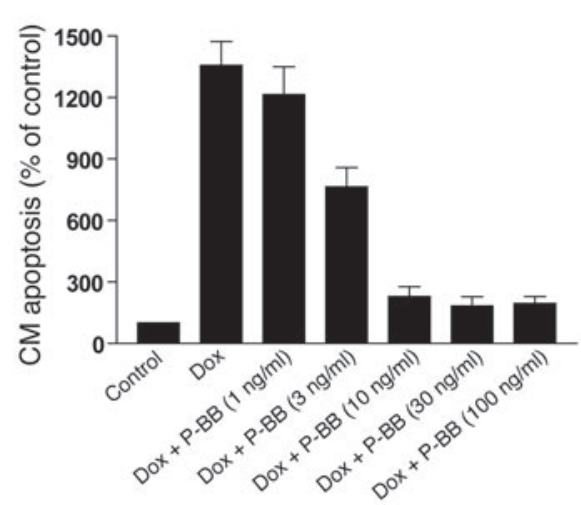

B

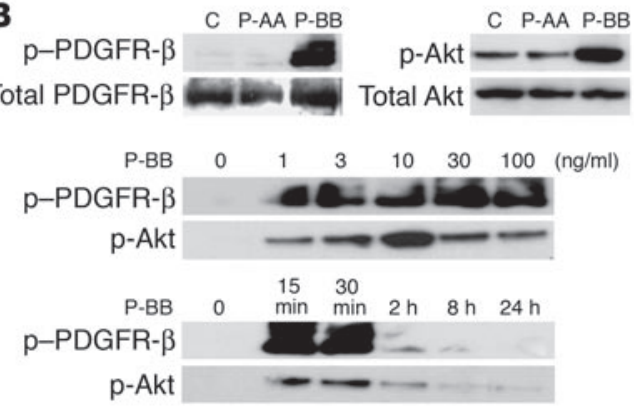

C
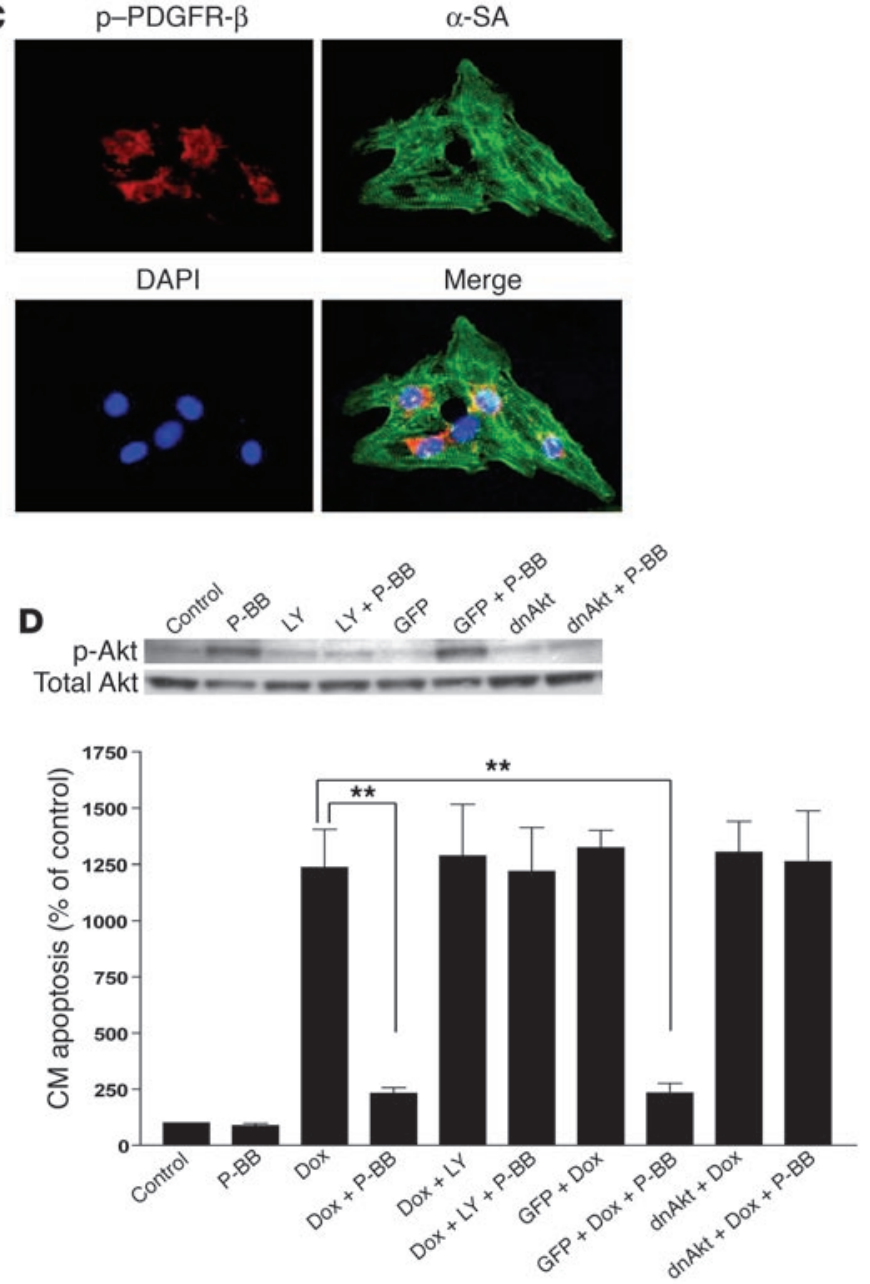

\section{Figure 2}

PDGF-BB prevents cardiomyocyte apoptosis via PI3K/Akt signaling. (A) Cardiomyocytes were treated with doxorubicin and PDGF-BB in concentrations as indicated $(\mathrm{ng} / \mathrm{ml})$. Apoptosis was determined by DNA fragmentation cytometry. (B) Immunoblotting of phospho-PDGFR- $\beta$ (p-PDGFR- $\beta$ ), total PDGFR- $\beta$, phospho-Akt (p-Akt), or total Akt in cardiomyocytes treated with $10 \mathrm{ng} / \mathrm{ml} \mathrm{PDGF-AA} \mathrm{or} \mathrm{PDGF-BB.} \mathrm{The} \mathrm{dose} \mathrm{(ng/ml)} \mathrm{and} \mathrm{time} \mathrm{(10}$ $\mathrm{ng} / \mathrm{ml}$ PDGF-BB) effects of phosphorylation of PDGFR- $\beta$ and Akt by PDGF-BB are also shown. (C) Immunofluorescence staining of PDGFR- $\beta$ phosphorylation and internalization in cardiomyocytes (yellow) treated with PDGF-BB. Red, p-PDGFR- $\beta$; green, $\alpha$-sarcomeric actinin ( $\alpha$-SA); blue, DAPI. (D) Immunoblotting of phospho-Akt and total Akt and apoptosis in cardiomyocytes treated with PDGF-BB, LY294002 (LY), and GFP adenovirus (Control) or dominant-negative Akt (dnAkt) adenovirus. ${ }^{* *} P<0.001$.

tions (Figure 3B). Protein extracted from the injected region was assayed by ELISA specific for human PDGF-BB. Human PDGF-BB was undetectable in rats with sham operation only, MI only, or MI with NFs (MI + NFs) only. Ten minutes after injecting 4 ng human PDGF-BB with or without peptide NFs, most of PDGF-BB was retained in the injected region in both groups $(79.5 \% \pm 18.3 \%$ in the group without peptide NFs and $91.8 \% \pm 6.4 \%$ in the group with peptide NFs; $P$ > 0.05; Figure 3B). Without peptide NFs, PDGF-BB rapidly disappeared from the injected sites after 24 hours, and only a negligible amount of PDGF-BB could be detected after 3 days. In contrast, with peptide NFs, PDGF-BB was retained at the injected sites; $16.1 \% \pm 2.4 \%$ of PDGF-BB remained at the targeted delivery sites after 14 days $(P<0.001$ for PDGF-BB with NFs versus PDGF-BB without NFs at 1-, 3-, and 14-day time points; Figure 3B). Immunohistochemical staining showed phosphorylation of PDGFR- $\beta$ at the injected sites by NF/PDGF-BB, but not by NFs or PDGF-BB alone or in the sham or MI only after 14 days (Fig- ure 3C). Immunofluorescence costaining confirmed sustained activation of PDGFR- $\beta$ in cardiomyocytes adjacent to the injected areas after 14 days (Figure 3D). Furthermore, to determine more quantitatively the difference in PDGFR- $\beta$ and Akt phosphorylation between groups, we used Western blotting to examine protein extracted from the injected sites and found sustained PDGFR- $\beta$ and Akt phosphorylation 1 and 14 days after injection of NFs with PDGF-BB (NF/PDGF-BB) (Figure 3E). Injection of PDGF-BB alone slightly induced PDGFR- $\beta$ and Akt phosphorylation after 1 day, but both disappeared by day 14 . These results demonstrate that injectable self-assembling peptide NFs can successfully deliver PDGF-BB into the myocardium, leading to prolonged activation of the PDGF signaling pathway in cardiomyocytes in vivo.

Injection of $N F / P D G F-B B$ preserves myocardial function. To examine the functional effects of injecting self-assembling peptide NF/ PDGF-BB for cardioprotection, we then performed a blinded and randomized study in 96 rats, with at least 6 animals in each experi- 
A

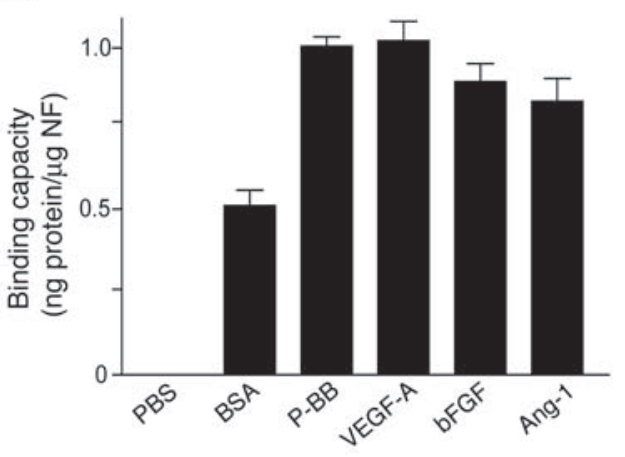

C
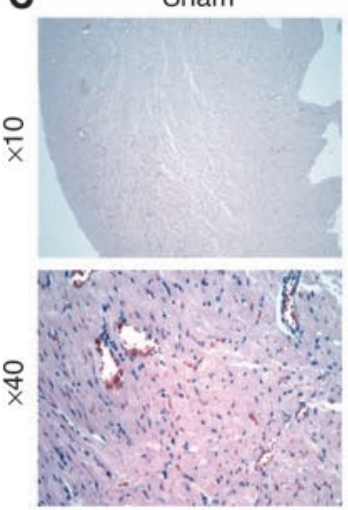

D

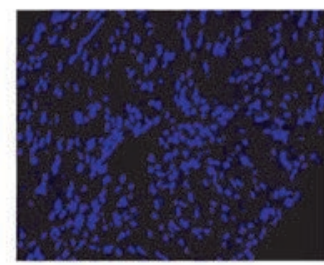

$\alpha-S A$

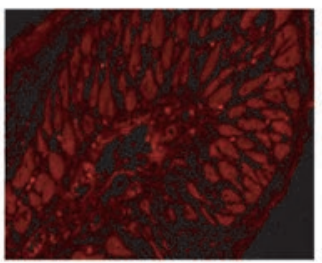

B

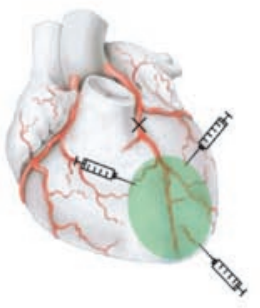

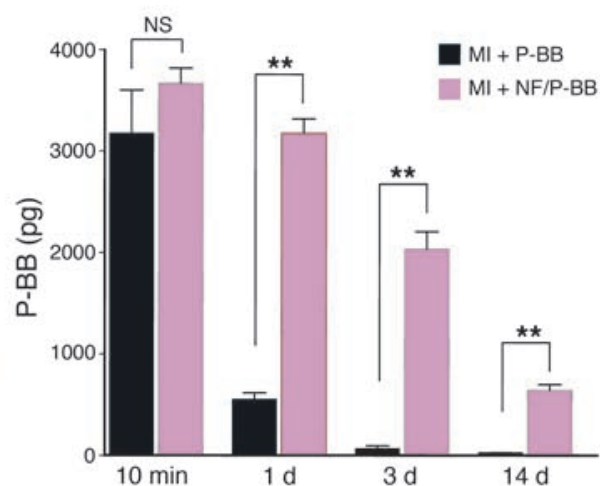

$\mathrm{MI}+\mathrm{NF}$
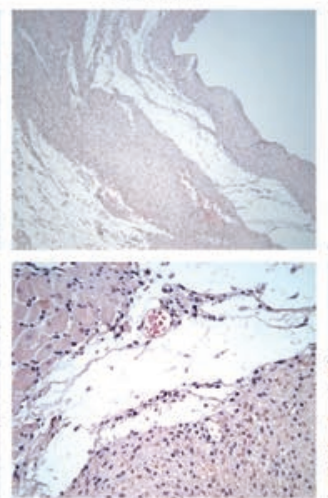

p-PDGFR- $\beta$

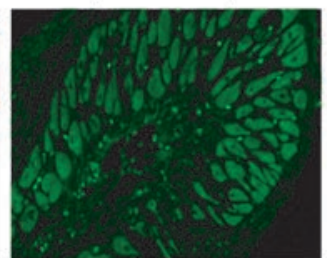

$\mathrm{MI}+\mathrm{NF} / \mathrm{P}-\mathrm{BB}$
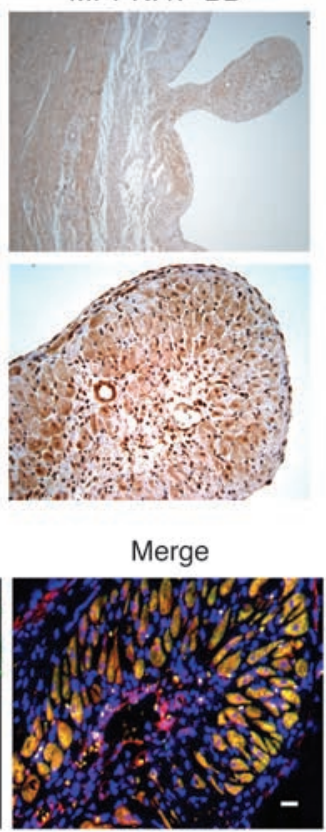

E

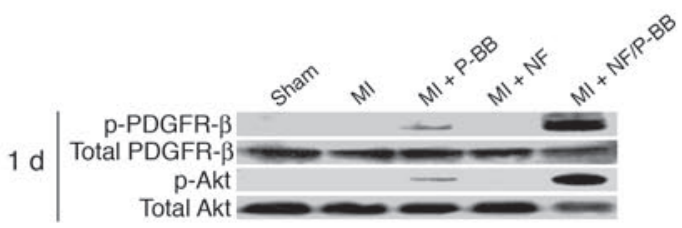

$$
14 \mathrm{~d} \mid \begin{array}{r}
\mathrm{p}-\mathrm{PDGFR}-\beta \\
\text { Total PDGFR- } \beta \\
\mathrm{p}-\mathrm{Akt} \\
\text { Total Akt }
\end{array}
$$

Figure 3

Controlled local myocardial delivery of PDGF-BB using injectable self-assembling peptide NFs. (A) PBS, BSA, PDGF-BB, VEGF-A, bFGF, or angiopoietin-1 (Ang-1) was embedded in NFs, and the binding capacity of NFs to individual protein was determined. (B) Rat left coronary artery was ligated to induce MI, followed with peptide NF injection in the border zones via 3 different directions. Myocardial protein was extracted from the injected areas, and retention of PDGF-BB, with or without NFs, was assessed using ELISA for human PDGF-BB. $n=6$ for each group; no human PDGF-BB was detected in control animals with NFs alone (data not shown). ${ }^{* \star} P<0.001$. (C) Immunohistochemistry of phospho-PDGFR- $\beta$ (brown) in sections from different groups 14 days after MI. (D) Immunofluorescence staining of phospho-PDGFR- $\beta$ in the myocardium after injection of NF/PDGF-BB. Shown is a 14-day section containing papillary muscle. Blue, DAPI; red, $\alpha$-sarcomeric actinin; green, phospho-PDGFR- $\beta$. Scale bar: $5 \mu \mathrm{m}$. (E) Myocardial proteins were extracted 1 and 14 days after Ml and subjected to Western blot analyses using antibodies against phospho-PDGFR- $\beta$, total PDGFR- $\beta$, phospho-Akt, and total Akt.

mental group. Two doses of PDGF-BB (50 and $100 \mathrm{ng} / \mathrm{ml}[\mathrm{P} 50$ and $\mathrm{P} 100])$ were selected for injection, estimated to deliver approximate PDGF-BB concentrations in the infarcted myocardium of 10 and
$20 \mathrm{ng} / \mathrm{ml}$, respectively, by injecting a total of $80 \mu \mathrm{l}$ (given at 3 different injection sites) of NF/PDGF-BB into $400 \mathrm{mg}$ of infarcted myocardium. Twenty-four hours after MI, left ventricular fractional 

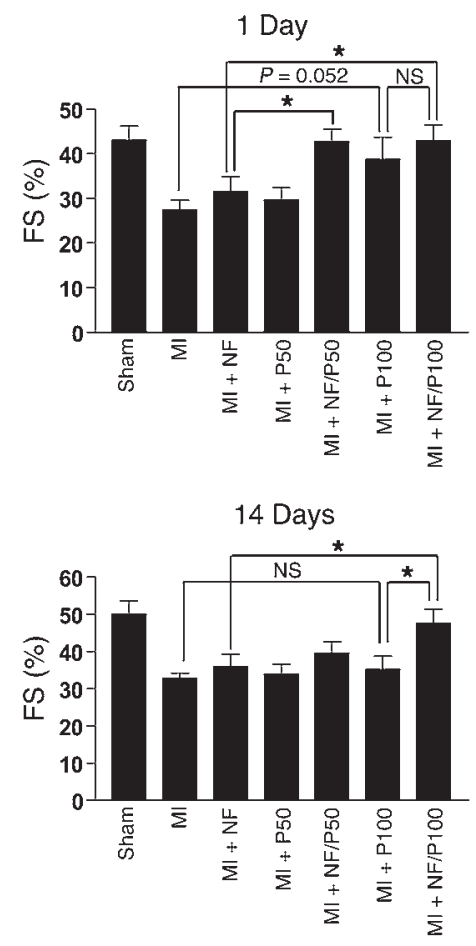

shortening decreased as anticipated compared with sham-operated myocardium $(43.1 \% \pm 7.7 \%$ in the sham group versus $27.5 \% \pm 5.6 \%$ in the MI group; $P<0.05)$, and injection of NFs or PDGF-BB alone did not significantly improve fractional shortening. However, in infarcted hearts with injection of NF/P50 or NF/P100, fractional shortening significantly improved $(42.7 \% \pm 7.1 \%$ and $43.0 \% \pm 8.4 \%$, respectively; $P<0.05$ for both versus MI or MI + NFs, Figure 4). At day 14 after infarction, improvement of fractional shortening was maintained in hearts that received NF/P100, but not in hearts that received NF/P50 $(50.0 \% \pm 8.7 \%$ in sham, $32.8 \% \pm 4.2 \%$ after MI, $39.6 \% \pm 7.9 \%$ in NF/P50, and $47.6 \% \pm 10.0 \%$ in NF/P100; $P<0.05$ for sham versus MI and NF/P100 versus MI or MI + NFs; Figure 4), implying dose-dependent cardioprotection by sustained release of PDGF-BB from the peptide NFs. Consistent with the improvement of fractional shortening after infarction, injection of NF/PDGF-BB also prevented cardiac dilation as measured by ventricular end-systolic and end-diastolic dimensions $(P<0.05$; Table 1$)$. Using trichrome staining, we did not observe an increase in fibrosis by injection of NF/PDGF-BB (data not shown), suggesting that injection of NF/PDGF-BB may not stimulate cardiac fibroblasts (25).

Injection of $N F / P D G F-B B$

decreases cardiomyocyte apoptosis and induces PI3K/Akt activation after infarction. To address whether preservation of cardiac systolic function by injection of peptide NF/PDGF-BB was accompanied by prevention of cardiomyocyte apoptosis, we examined tissues using immunofluorescence costaining of cleaved caspase- 3 and $\alpha$-sarcomeric actinin (Figure $5 \mathrm{~A})$. Injection of peptide NFs

\section{Figure 4}

Injection of NF/PDGF-BB preserves myocardial contractility and prevents dilation after infarction. Left ventricular fractional shortening (FS) 1 and 14 days after MI was measured using echocardiography. Two doses of PDGF-BB were used, P50 and P100, for injection with or without NFs. $n \geq 6$ in each group; ${ }^{\star} P<0.05$.

with 50 or $100 \mathrm{ng} / \mathrm{ml}$ PDGF-BB, but not NFs or PDGF-BB alone, dramatically decreased caspase- 3 activation after 1 day $(P<0.001$ for 50 or $100 \mathrm{ng} / \mathrm{ml}$ PDGF-BB with NFs versus MI or MI + NFs; Figure 5B). After 14 days, injection of NF/P100 reduced apoptosis more than injection of NF/P50, although both treatments significantly reduced apoptosis compared with controls $(0.5 \% \pm 0.2 \%$ in sham, $12.8 \% \pm 1.1 \%$ in MI, $7.2 \% \pm 0.8 \%$ in NFs with $50 \mathrm{ng} / \mathrm{ml}$ of PDGF-BB, $P<0.01$ versus MI; and $3.1 \% \pm 0.4 \%$ in NFs with 100 $\mathrm{ng} / \mathrm{ml}$ of PDGF-BB, $P<0.001$ versus MI; Figure $5 \mathrm{~B}$ ). Furthermore, we demonstrated activation of Akt in the myocardium by injection of NF/PDGF-BB but not when NFs or PDGF-BB alone was injected (Figure 6A), showing that this strategy induces survival signaling in the myocardium in vivo (26-28). With addition of the PI3K-specific inhibitor LY294002 $(50 \mu \mathrm{M})$ in peptide NF/PDGF$\mathrm{BB}$, we found that Akt activation was blocked and improvement of fractional shortening by injection of NF/PDGF-BB was also abolished (Figure 6A), implying that PI3K/Akt signaling may play a role in the cardioprotective effect of injecting peptide NF/ PDGF-BB. Moreover, as multiple myocardial cells may be targets for PDGF-BB, we examined Akt phosphorylation in cardiomyocytes, endothelial cells, VSMCs, and fibroblasts after injection of NF/PDGF-BB using immunofluorescence costaining of phosphoAkt and cell-specific markers. We found that Akt phosphorylation was induced in cardiomyocytes after 1 and 14 days and also in endothelial cells after 14 days of injection (Figure 6B). However, we were not able to detect Akt phosphorylation in VSMCs or fibroblasts. These results imply that cardiomyocytes are the main targets for PDGF-BB after injection of NF/PDGF-BB.

Injection of $N F / P D G F-B B$ decreases infarct size after $I / R$ injury. To investigate whether injection of NF/PDGF-BB may also benefit the heart from ischemia/reperfusion (I/R) injury, we conducted another blinded and randomized experiment in 52 rats to examine infarct size after 60-minute ischemia and 24-hour reperfusion $(n=10$ in sham and I/R alone groups; $n=16$ in I/R + NFs alone and I/R + NF/PDGF-BB groups). Injection of NF/P100, but not NFs alone, improved ventricular fractional shortening after 24 hours of reperfusion $(50.3 \% \pm 2.0 \%$ in sham, $36.1 \% \pm 2.0 \%$ in $\mathrm{I} / \mathrm{R}$ only, $41.9 \% \pm 2.4 \%$ in $\mathrm{I} / \mathrm{R}+\mathrm{NFs}$, and $51.3 \% \pm 2.1 \%$ in $\mathrm{I} / \mathrm{R}+$

\section{Table 1}

End-diastolic dimension and end-systolic dimension by echocardiography 1 and 14 days after infarction

$\begin{array}{ccccccccc} & & \text { Sham } & \text { MI } & \text { MI + NF } & \text { MI + P50 } & \text { MI + NF/P50 } & \text { MI + P100 } & \text { MI + NF/P100 } \\ & n & 6 & 6 & 7 & 7 & 6 & 6 & 6 \\ 1 \mathrm{~d} & \text { EDD } & 5.1 \pm 0.4 & 6.0 \pm 0.9 & 5.6 \pm 0.6 & 5.7 \pm 0.6 & 5.3 \pm 0.5 & 5.8 \pm 0.7 & 5.2 \pm 0.7 \\ & \text { ESD } & 2.9 \pm 0.3 & 4.4 \pm 0.7 & 3.8 \pm 0.7 & 4.0 \pm 0.7 & 3.2 \pm 0.5^{\mathrm{A}} & 3.6 \pm 0.8 & 3.0 \pm 0.8 \\ & n & 7 & 7 & 7 & 8 & 8 & 7 & 8 \\ 14 \mathrm{~d} & \text { EDD } & 5.6 \pm 0.5 & 7.2 \pm 1.1 & 6.5 \pm 1.3 & 6.5 \pm 0.9 & 6.4 \pm 1.0 & 6.5 \pm 0.7 & 5.7 \pm 0.4^{\mathrm{B}} \\ & \text { ESD } & 2.5 \pm 0.7 & 4.8 \pm 1.1 & 4.2 \pm 1.3 & 4.4 \pm 1.0 & 3.8 \pm 0.9 & 4.0 \pm 1.1 & 2.8 \pm 1.1^{\mathrm{B}}\end{array}$

EDD, end-diastolic dimension; ESD, end-systolic dimension. ${ }^{\mathrm{A} P}<0.05$ compared with $\mathrm{MI}+\mathrm{P} 50$; ${ }^{\mathrm{B} P}<0.05$ compared with $\mathrm{Ml}+\mathrm{P} 100$. 
A

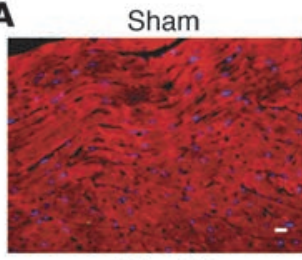

$\mathrm{MI}+\mathrm{NF}$

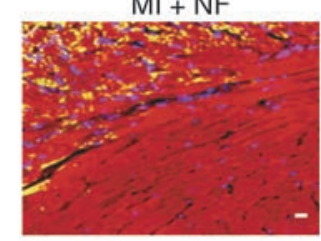

B
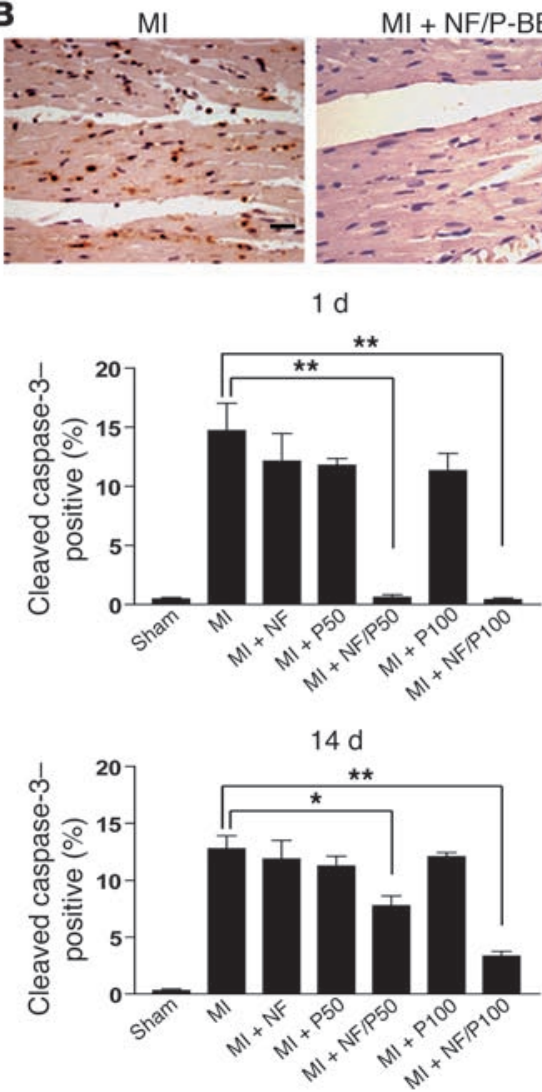

NF/PDGF-BB; $P<0.05$ for I/R + NF/PDGF-BB versus I/R only or $\mathrm{I} / \mathrm{R}+\mathrm{NFs}$; Figure $7 \mathrm{~A})$. Infarct size, represented by percent of area at risk, was also decreased by injection of NF/PDGF-BB $(0 \%$ in sham, $40.0 \% \pm 2.3 \%$ in $\mathrm{I} / \mathrm{R}$ only, $38.5 \% \pm 2.2 \%$ in $\mathrm{I} / \mathrm{R}+\mathrm{NFs}$, and $27.8 \% \pm 2.4 \%$ in $\mathrm{I} / \mathrm{R}+\mathrm{NF} / \mathrm{PDGF}-\mathrm{BB}$; $P<0.01$ for $\mathrm{I} / \mathrm{R}+\mathrm{NF} / \mathrm{PDGF}-$ $\mathrm{BB}$ versus I/R only or I/R + NFs; Figure $7 \mathrm{~B})$. As expected, the area at risk, represented by the percentage volume of the left ventricle, was similar in I/R only, I/R + NFs, and I/R + NF/PDGF-BB groups (Figure $7 \mathrm{C}$ ), indicating that the location of coronary ligation was similar among the groups. We also generated a new randomized and blinded experiment to study the effects of PDGF-BB alone on infarct size ( 6 rats for I/R only and 6 rats for I/R + PDGF-BB) and did not observe statistical difference between these 2 groups $(P=0.645$; data not shown). Taken together, these results demonstrate that injection of NF/PDGF-BB may preserve myocardial function after infarction and $\mathrm{I} / \mathrm{R}$ injuries.

\section{Figure 5}

Injection of NF/PDGF-BB decreases cardiomyocyte death after infarction. (A) Immunofluorescence staining of cleaved caspase-3 in cardiomyocytes (yellow areas) was shown in sections from 1 day after injection of NFs with or without P100. Blue, DAPI; red, $\alpha$-sarcomeric actinin; green, cleaved caspase-3. (B) Immunohistochemistry of cleaved caspase-3 (shown in brown) was used to quantify the amount of cleaved caspase-3-positive cells in the border zones 1 and 14 days after MI. Bar graphs show percent of positive cells in sections, counted without knowledge of treatment groups. ${ }^{*} P<0.01$; ${ }^{* *} P<0.001$. Scale bar: $5 \mu \mathrm{m}$.

Injection of NF/PDGF-BB does not increase myocardial cell proliferation, neovascularization, regional blood flow, or inflammation after infarction. Since PDGF-BB is a potent mitogen for many cells, we studied cell proliferation induced by PDGF-BB delivery in the infarcted myocardium using BrdU and Ki67 to label proliferative cells. Active cell proliferation could be detected in the positive control, rat small intestine, by BrdU staining (data not shown). In contrast, less than $0.5 \%$ cells were BrdU positive in the periinfarct myocardium after 1 (data not shown), 14 (Figure 8A), and 28 (data not shown) days, and there were no differences in number of BrdU-positive cells among all of the study groups (Figure 8A). Similar results were found using Ki67 staining (Figure 8B). Interestingly, costaining Ki67 and cell-specific markers revealed that cell proliferation occurred only in endothelial cells and fibroblasts, but not in cardiomyocytes or VSMCs, and there were no differences in numbers of Ki67-positive cells between groups (Figure 8B). Consistent with these results, using $\left[{ }^{3} \mathrm{H}\right]$ thymidine incorporation assay, we found PDGF-BB did not induce DNA synthesis in cultured rat cardiomyocytes or cardiac fibroblasts but did increase DNA synthesis in human aortic smooth muscle cells (data not shown). These results suggest that injection of NF/PDGF-BB does not induce cell proliferation in the ischemic myocardium.

We then tested whether injection of NF/PDGF-BB could enhance neovascularization in the ischemic myocardium because we have previously found that injection of NFs alone in normal myocardium led to neovascularization within the NFs (29). The overall capillary (endothelial cell staining) and arterial (VSMC staining) densities (vessel number per millimeter ${ }^{2}$ ) in the periinfarct area were not changed by injection of NFs, with or without PDGF-BB, after 14 and 28 days of infarction (Figure 9, A and B). Vascular diameters were also not different between groups (data not shown). Consistent with these data, using fluorescent microspheres, we found that regional blood flow in the myocardium was decreased 14 days after infarction, but not changed by injection of NF/PDGF-BB, NFs alone, or PDGF-BB alone (Figure 9C). We also examined whether injection of NFs with or without PDGF-BB could trigger an inflammatory response and found that there was no increase in neutrophil or monocyte/macrophage infiltration after injection compared with MI without injection of NFs (Figure 9, A and B). Together these data suggest that improvement of cardiac function by NF/PDGF-BB injection may not result from improvement of blood supply.

\section{Discussion}

Given the rapid loss of cardiomyocytes after ischemic injury, promoting cardiomyocyte survival is an efficient strategy for preserving viable myocardium. The principal findings of this study are that PDGF-BB is an endothelium-secreted paracrine factor with 
A
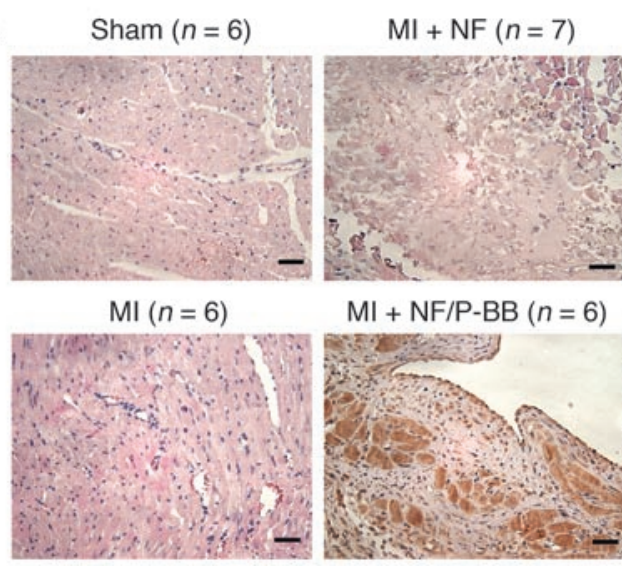

$\mathrm{MI}+\mathrm{NF} / \mathrm{P}-\mathrm{BB}(n=6)$
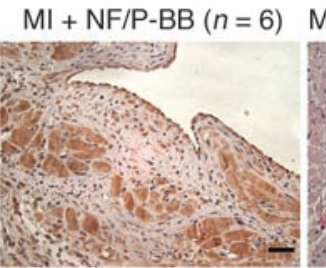

B

Cell type

$\mathrm{CM}$
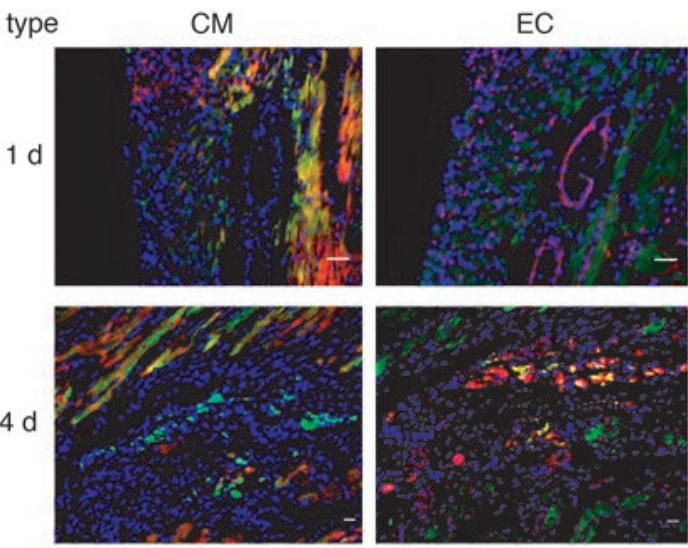

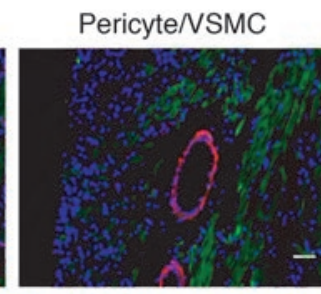

$\mathrm{MI}+\operatorname{NF} / \mathrm{LY}(n=7)$

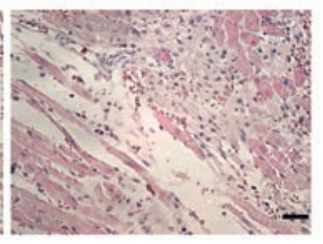

$\mathrm{MI}+\mathrm{NF} / \mathrm{LY} / \mathrm{P}-\mathrm{BB}(n=9)$
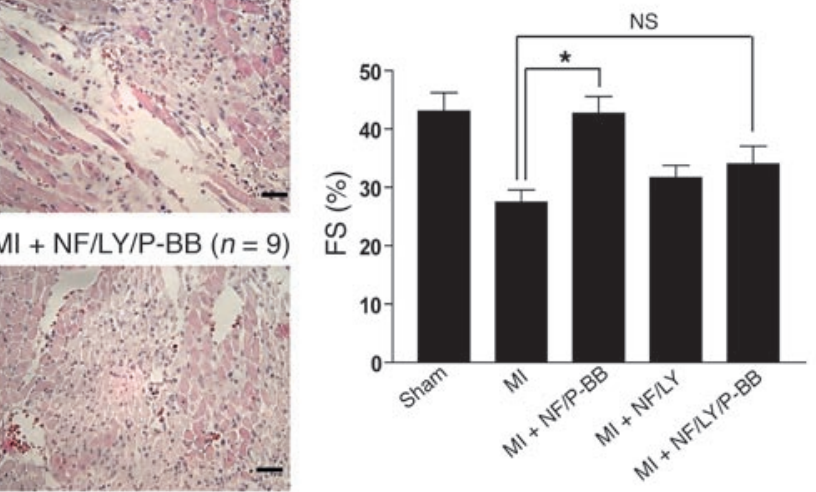

FB
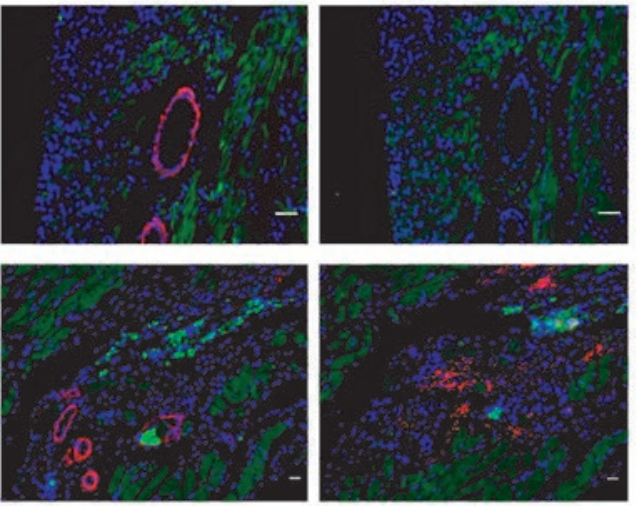

Figure 6

Injection of NF/PDGF-BB preserves cardiac contractility via PI3K/Akt signaling. (A) Immunohistochemistry of phospho-Akt (brown) from rat sections 1 day after injection of NFs with or without PDGF-BB or LY294002. Scale bars: $10 \mu \mathrm{m}$. Left ventricular fractional shortening 1 day after MI was also measured using echocardiography. ${ }^{*} P<0.05$. (B) Immunofluorescence costaining of phospho-Akt (green) and cell-specific markers (red) for cardiomyocytes (tropomyosin), endothelial cells (isolectin), pericytes/VSMCs ( $\alpha$-SMA), and fibroblasts (vimentin) on series of sections 1 and 14 days after injection of NF/PDGF-BB. Note Akt phosphorylation in cardiomyocytes (1- and 14-day) and endothelial cells (14-day). Scale bars: $20 \mu \mathrm{m}$ in the 1-day sections and $5 \mu \mathrm{m}$ in the 14-day sections.

direct antiapoptotic effects in cardiomyocytes and that this prosurvival signal can be delivered into the infarcted myocardium for cardioprotection in a highly controlled manner using injectable self-assembling peptide NFs.

An obvious advantage of local delivery for molecular therapy is that adverse effects of a therapy may be more constrained to the local environment. However, many current methods of local delivery lack stringent controls that are valuable for therapy. For example, simple injection of a factor is limited by the rapid diffusion of proteins away from the injection site. On the other hand, prolonged overexpression by gene therapy vectors can lead to excessive protein production and an undesired outcome. Local control of protein concentration is potentially critical. For example, Ozawa et al. reported that the microenvironmental amount of VEGF, rather than the overall dose, may determine whether normal angiogenesis or aberrant hemangiomas would be induced after VEGF gene transfer (30).

\section{Figure 7}

Injection of NF/PDGF-BB decreases infarct size after I/R injury. (A) Left ventricular fractional shortening after 60-minute ischemia and 24-hour reperfusion with or without injection of NF/PDGF-BB was measured using echocardiography. Hearts were then harvested and subjected to measurement of infarct size and area at risk (B and $\mathbf{C})$. ${ }^{*} P<0.05$.

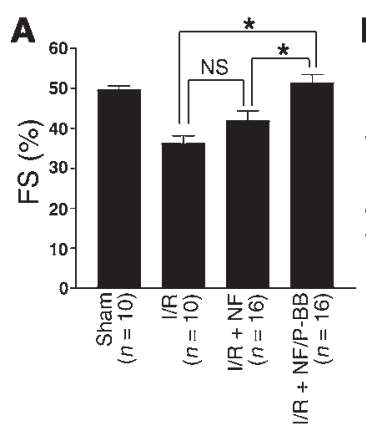

B

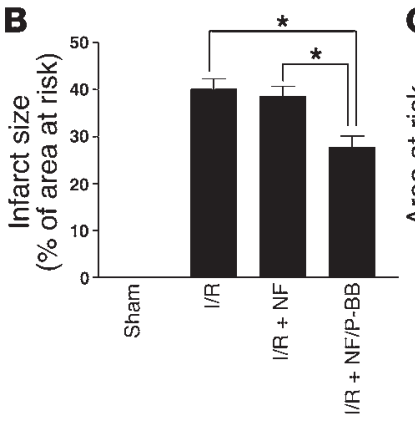

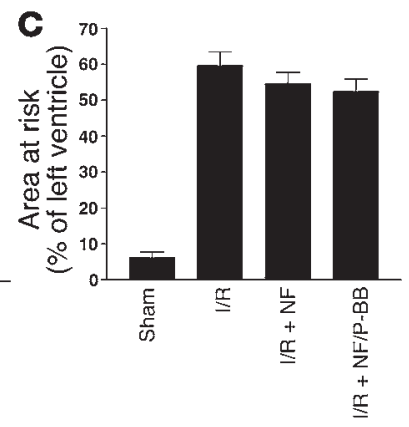


A

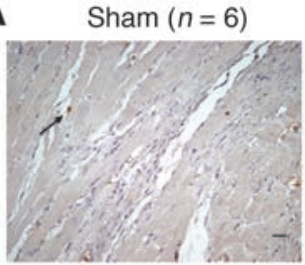

$\mathrm{MI}+\mathrm{NF}(n=7)$

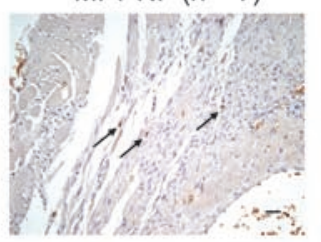

$\mathrm{Ml}+\mathrm{NF} / \mathrm{P}-\mathrm{BB}(n=6)$
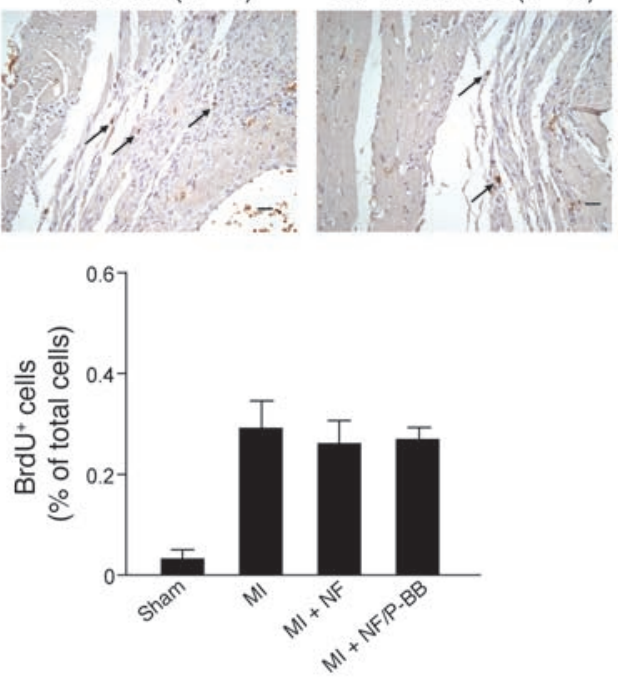

C
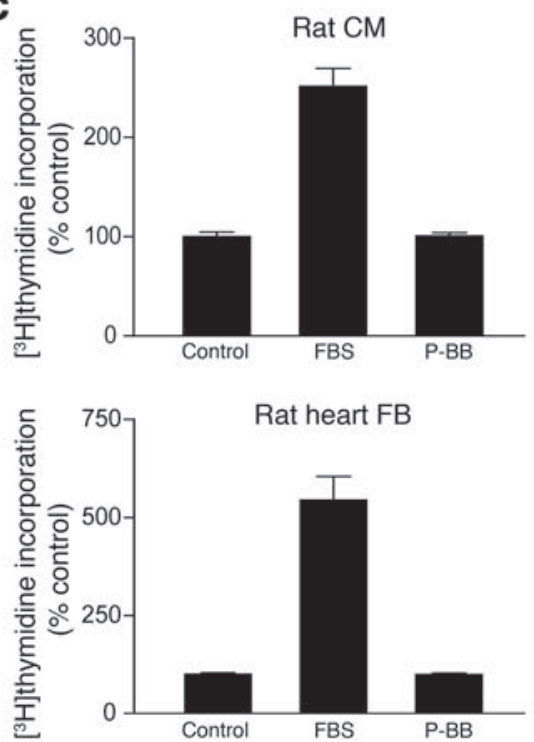

B

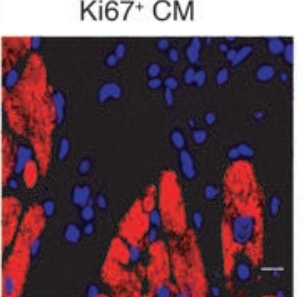

Ki67+ VSMC
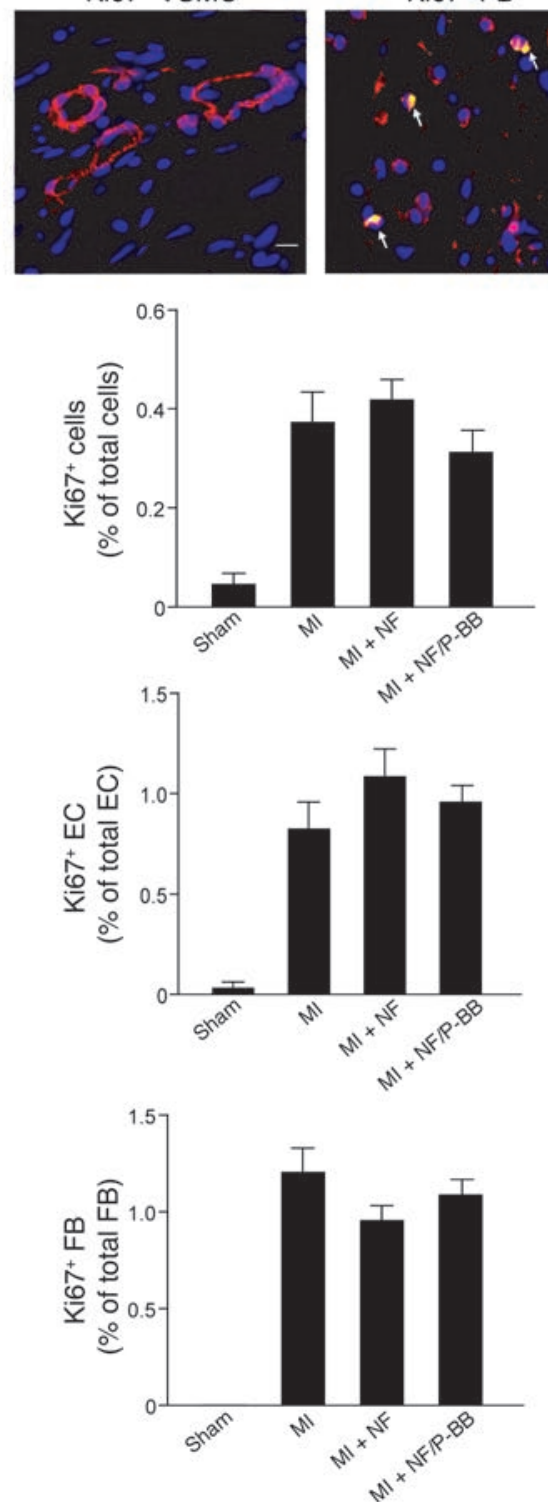

Figure 8

Injection of NF/PDGF-BB does not increase cell proliferation after infarction. (A) Immunohistochemistry of BrdU was used to measure total cell proliferation at the periinfarct areas after 14 days of MI. Also shown are the percentages of BrdU-positive cells (arrows) in the different groups ( $n \geq 6$ in each group; in total, $\sim 300$ cells counted in each animal). Scale bars: 5 um. (B) Immunofluorescence costaining of Ki67 with cell-specific markers was also used to examine cell proliferation 14 days after Ml. Red, cell markers, including tropomyosin for cardiomyocytes, isolectin for endothelial cells, $\alpha$-SMA for pericytes/VSMC, and vimentin for fibroblasts; yellow, Ki67 cells and cell markers; blue, DAPI. Arrows indicate Ki67-positive cells. Scale bars: $5 \mu \mathrm{m}$. Also shown are the percentages of Ki67-positive cells in total cell population and in individual endothelial and fibroblast population. (C) DNA synthesis measurement using $\left[{ }^{3} \mathrm{H}\right]$ thymidine incorporation was performed to study the mitogenic effect of 10 $\mathrm{ng} / \mathrm{ml}$ PDGF-BB and 10\% FBS on cultured rat cardiomyocytes and cardiac fibroblasts.
Self-assembling peptide NFs are unique biomaterials with the potential for assisting tissue regeneration. Previously we have found that injection of self-assembling NFs into the nonischemic myocardium may induce rapid endothelial and myocyte-like precursor cell recruitment within 1-2 weeks and mature vasculature in the microenvironments after 4 weeks (29). In this study, we sought to explore the possibility of using these injectable NFs for controlled delivery of therapeutic proteins into the ischemic heart. Interestingly, we did not observe similar enhanced neovascularization in the NF microenvironments in the present study, possibly due to differences between nonischemic myocardium and the more inflammatory environment of ischemic myocardium. However, our results show that this strategy is highly effective for delivery of PDGF-BB in the early period after infarction; even after 14 days, $16 \%$ of PDGF-BB still remained in the injected sites. Compared with injection of PDGF-BB alone, which was rapidly eliminated after injection, NF/PDGF-BB injection achieved sustained delivery of PDGF-BB inside the myocardium. Importantly, PDGF-BB embedded within the NFs remained biologically active after 14 days of injection, inducing phosphorylation of PDGFR- $\beta$ 
A
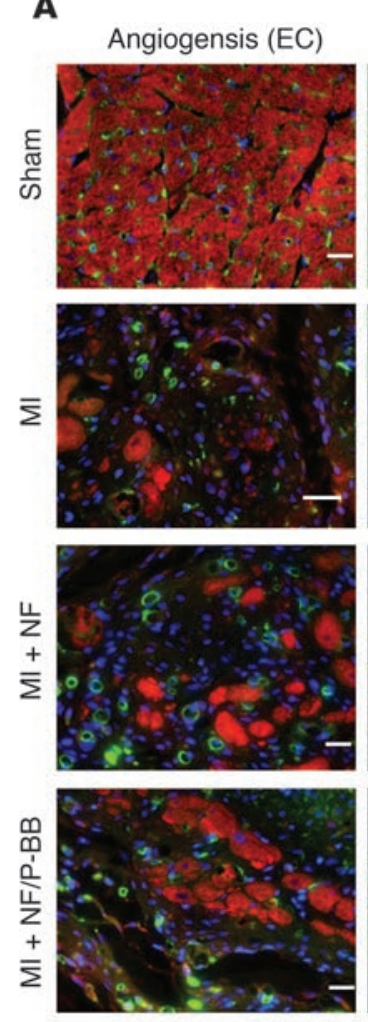
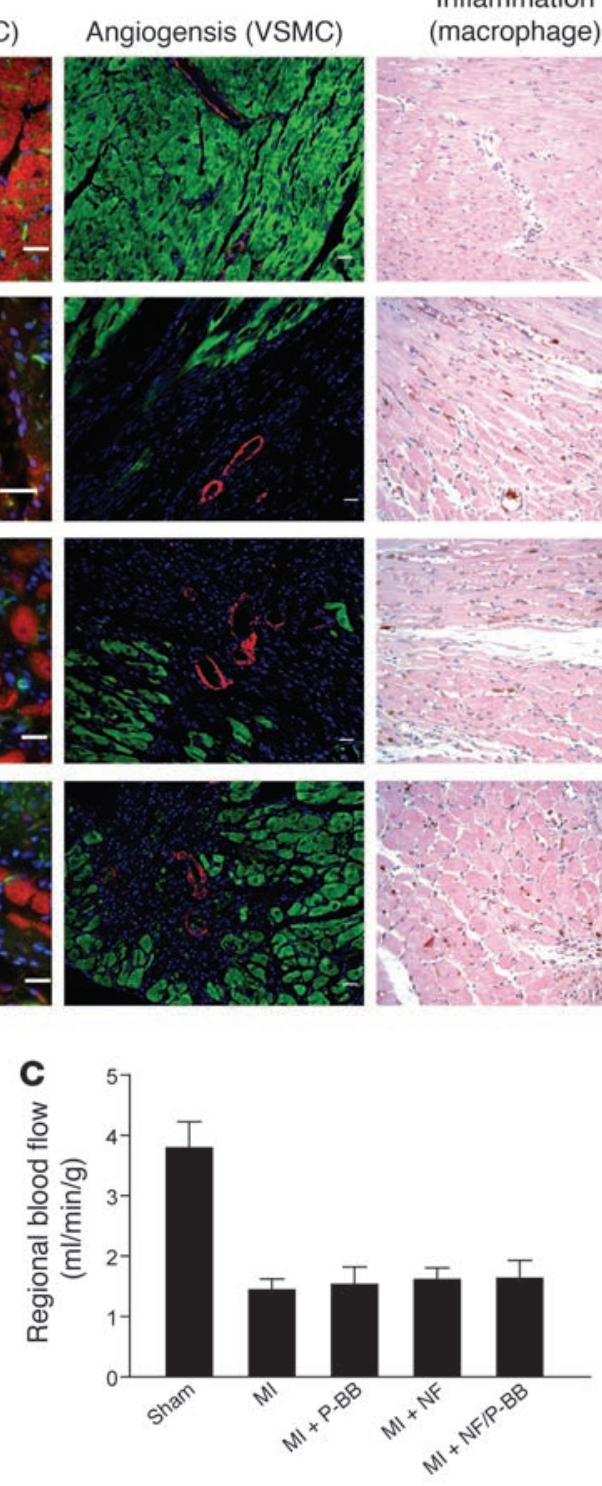
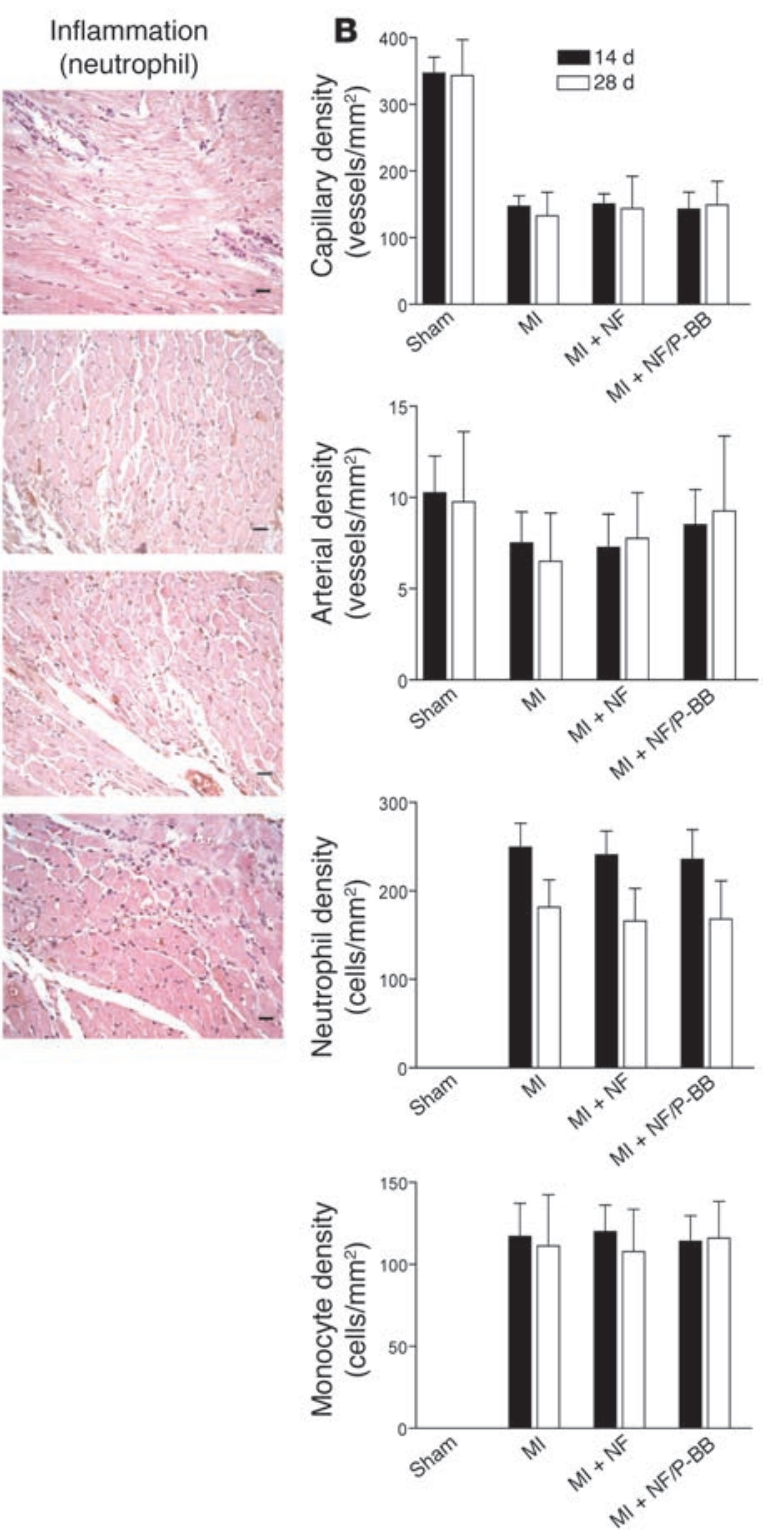

Figure 9

Injection of NF/PDGF-BB does not increase myocardial angiogenesis/arteriogenesis, local blood flow, or inflammatory reaction after infarction. (A) Immunofluorescence staining of endothelial cells (isolectin, green) and VSMCs ( $\alpha$-SMA, red), counterstained with $\alpha$-sarcomeric actinin and DAPI (blue) was used to evaluate capillaries and arteries, respectively, after 14 and 28 (data not shown) days of MI with or without injection of NF/PDGF-BB. Immunohistochemistry for macrophages (anti-mac3) and neutrophils (anti-neutrophil) was also performed to examine inflammatory reaction after 14 and 28 (data not shown) days of MI. Scale bars: $10 \mu \mathrm{m}$ in endothelial cell stains, $20 \mu \mathrm{m}$ in VSMC stains, and $5 \mu \mathrm{m}$ in macrophage and neutrophil stains. Vascular and inflammatory cell densities were determined using fluorescence and light microscopy, and the results are shown in B ( $n \geq 6$ in each group; in total, $\sim 200$ capillaries, $\sim 50$ arteries, $\sim 300$ macrophages, and $\sim 300$ neutrophil cells counted in each animal). (C) A separate blinded and randomized study with 6 rats in each group was performed to measure local myocardial blood flow after 14 days of MI using fluorescent microspheres. No impact of PDGF-BB delivery on local blood flow was detected.

and Akt in cardiomyocytes through the entire layer of myocardi$\mathrm{um}$ at the injected areas and, occasionally, even into the papillary muscles (Figure 3, C-E). Given the transient PDGFR- $\beta$ and Akt phosphorylation in cardiomyocytes by PDGF-BB in vitro, the profile of PDGFR- $\beta$ and Akt phosphorylation seems different from the sustained activation in vivo. Although we do not know the exact mechanisms for this difference, it is possible that NFs serve as a reservoir for slow release of PDGF-BB and that there is serial activation of cardiomyocytes by PDGF-BB.
We then showed the impact of PDGF-BB retention, as injection of NF/PDGF-BB improved cardiac systolic function after 1 and 14 days of infarction in a dose-dependent manner; NF/P100 seemed more beneficial than NF/P50. Chamber dilation was also improved by NF/PDGF-BB injection over PDGF-BB injection alone. However, it should be noted that there may be a dosedependent effect after injection of PDGF-BB alone. The $P$ value for comparison of MI + NF/P100 vs. MI + P100 was 0.26 at the 1 -day time point for fractional shortening; the $P$ value for comparison of 
$\mathrm{MI}+\mathrm{NF} / \mathrm{P} 100$ versus MI + P100 was 0.018 for the 14 -day time point. Moreover, the $P$ value for comparison between MI + P100 and MI only was 0.052 at the 1 -day time point, and the $P$ value of comparison between $\mathrm{MI}+\mathrm{P} 100$ and MI only was 0.19 at the 14-day time point (Figure 4A). These results suggest that injection of PDGF-BB at high doses without NFs may be beneficial in the early period, but sustained improvement at 14 days requires NF delivery. These results also suggest that using NFs for controlled release of PDGF$\mathrm{BB}$, even without combining other factors such as VEGF-A and angiopoietin-1 (31), may be effective for myocardial protection.

Mechanistically, we confirmed that cardiomyocyte death was decreased, and the improvement of cardiac systolic function may depend on PI3K/Akt signaling. However, since injection of NF/P50 decreased cardiomyocyte death after 14 days but did not improve ventricular performance at the same time point, other mechanisms underlying functional improvement may exist. We examined other possible mechanisms including cell proliferation, angiogenesis/ arteriogenesis, regional blood flow, and inflammation after injection. BrdU staining showed that overall cell proliferation at the periinfarct area was not different among all groups at 1, 14, and 28 days after MI. Costaining for Ki67, another proliferation marker, with cell-specific antigens also revealed no increase in cell proliferation in cardiomyocytes, endothelial cells, pericytes/VSMCs, or fibroblasts after injection of NF/PDGF-BB. Consistent with the in vitro data showing that PDGF-BB did not increase DNA synthesis in cultured cardiomyocytes or cardiac fibroblasts, these data indicate that controlled delivery of PDGF-BB does not induce myocardial cell proliferation after infarction. Although the reasons why delivery of PDGF-BB with NFs did not induce any cell proliferation after MI remain to be explored, these results indicate that continuous release of PDGF-BB from NFs does not stimulate cell proliferation in the ischemic myocardium. Furthermore, injection of NFs, with or without PDGF-BB, did not increase the inflammatory response in the injured myocardium. Finally, in a separate experiment, injection of NF/PDGF-BB 60 minutes following ischemia also preserved cardiac systolic function and reduced infarct size after 24 hours of reperfusion. These results suggest that this approach may potentially benefit patients with MI, but it is important to recognize that our study does not define the therapeutic window after infarction for successful therapy. Additional studies should be performed to determine this therapeutic window, both in rats and in other species.

Many current strategies for myocardial protection and repair are challenged by poor implanted cell survival or rapid diffusion of factors away from injection areas or by the delivery technique itself, such as the use of stiff percutaneous transaortic catheters for cardiac interventions. The requirement of direct intramyocardial injection may limit application of any therapy relative to the potential feasibility of systemic therapy. However, self-assembling peptide NFs represent a new approach for sustained delivery of candidate factors into the myocardium. Successful reconstruction of the myocardium will require tightly regulated signals that can potentially be controlled by modifications of the peptides (32) and candidate factors to be delivered. Self-assembling peptide NFs may therefore be able to provide a local microenvironment to deliver a cardiac preservation signal, as well as additional signals that promote cardiac repair.

\section{Methods}

Myocardial cell culture. Rat neonatal cardiomyocytes (1-2 days old) and adult cardiac endothelial cells and fibroblasts were isolated from Sprague-Dawley rats (Charles River Laboratories and Harlan) as previously described (15).
Cardiomyocyte apoptosis assays. Cardiomyocytes were plated at a density of $1.4 \times 10^{5}$ cells $/ \mathrm{cm}^{2}$ overnight, cultured in serum-free DMEM for 24 hours, and then subjected to $10 \mu \mathrm{M}$ chelerythrine, $1 \mu \mathrm{M}$ doxorubicin (both from Sigma-Aldrich), or $0.2 \mathrm{mM} \mathrm{H}_{2} \mathrm{O}_{2}$ plus $100 \mathrm{ng} / \mathrm{ml} \mathrm{TNF- \alpha}$ (PeproTech) for another 24 hours with or without treatment with human PDGF-BB (PeproTech). In coculture experiments, before plating of cardiomyocytes, cardiac endothelial cells or fibroblasts were cultured until subconfluent. TUNEL staining was performed using a TUNEL staining kit (Roche Diagnostics Corp.). For DNA fragmentation experiments, cells were trypsinized, fixed with $80 \%$ ethanol at $-20^{\circ} \mathrm{C}$ for 2 hours, incubated in $0.1 \mathrm{mg} / \mathrm{ml} \mathrm{RNase}$ (Sigma-Aldrich) at $37^{\circ} \mathrm{C}$ for 30 minutes, stained with $0.1 \mathrm{mg} / \mathrm{ml}$ propidium iodide (Sigma-Aldrich) for 10 minutes, and then subjected to flow cytometry (Cytomics FC 500; Beckman Coulter). The Annexin- $V$ cell sorting method was performed using an apoptosis assay kit (Vybrant Apoptosis Assay Kit \#3; Molecular Probes, Invitrogen Corp.) following the manufacturer's instructions. The dosages for neutralizing antibodies were $0.4 \mu \mathrm{g} / \mathrm{ml}$ anti-PDGF-BB, $2 \mu \mathrm{g} / \mathrm{ml}$ anti-PDGFR- $\beta, 2 \mu \mathrm{g} /$ $\mathrm{ml}$ anti-PDGF-AA, and $20 \mu \mathrm{g} / \mathrm{ml}$ anti-PDGFR- $\alpha$ (all from Sigma-Aldrich except anti-PDGF-AA from R\&D Systems), and the dosage of PDGF-BB was $10 \mathrm{ng} / \mathrm{ml}$ unless otherwise indicated. Adenoviral transfection was performed as previously described by Fujio and Walsh (33). Each experiment was performed in triplicate and repeated at least 3 times using different primary cell preparations.

Western blot analysis. Whole-cell extracts were collected using lysis buffer containing 1\% (wt/vol) SDS, $50 \mathrm{mM}$ Tris-Cl ( $\mathrm{pH} 7.4$ ), 5 mM EDTA supplemented with $4 \times$ sample buffer (Invitrogen Corp.) and proteinase inhibitor cocktail (Sigma-Aldrich) at 1:500 dilution. The following antibodies were used: anti-PDGFR- $\beta$ (Santa Cruz Biotechnology Inc.), anti-phosphoPDGFR- $\beta$, anti-Akt, anti-phospho-Akt, anti-phospho-ERK, anti-phosphop38, and anti-jun $\mathrm{N}$-terminal kinase (all from Cell Signaling Technology).

Immunocytochemistry. For immunocytochemical staining of PDGFR- $\beta$ activation, cardiomyocytes were prepared using the same method as described above using anti-phospho-PDGFR- $\beta$ antibody (Cell Signaling Technology).

Protein binding assay of self-assembling NFs. NFs were prepared as previously described (15), and PBS or 100 ng of BSA (Sigma-Aldrich), PDGF-BB (PeproTech), VEGF-A, bFGF, or angiopoietin-1 (all from R\&D Systems) was embedded in NFs. NFs were incubated with PBS $(100 \mu \mathrm{l})$ at $37^{\circ} \mathrm{C}$ for 3 hours. Supernatant (PBS after incubation) was collected and subjected to Bradford protein assay. In parallel experiments, the same amount of proteins was added in PBS but not embedded within NFs, in order to demonstrate that the experimental binding conditions were at equilibrium.

MI and injection of peptide NFs. All animal protocols were approved by the Harvard Medical School Standing Committee on Animals. MI was produced in approximately 250-g male Sprague-Dawley rats (Charles River Laboratories and Harlan). Briefly, rats were anesthetized by pentobarbital and, following tracheal intubation, the hearts were exposed via left thoracotomy. The left coronary artery was identified after pericardiotomy and was ligated by suturing with 6-0 prolene at the location approximately 3 $\mathrm{mm}$ below the left atrial appendix. For the sham operation, suturing was performed without ligation. Peptide NFs (peptide sequence AcN-RARADADARARADADA-CNH $\mathrm{CH}_{2}$ from SynPep Corp.) with or without 50 or 100 $\mathrm{ng} / \mathrm{ml}$ human PDGF-BB (PeproTech) and/or $50 \mu \mathrm{M}$ LY294002 (Calbiochem) were dissolved in $295 \mathrm{mM}$ sucrose and sonicated to produce a $1 \%$ solution for injection. A total of $80 \mu$ l of self-assembling peptide NFs was injected into the infarcted border zone through 3 directions (equal amount for each injection) immediately after coronary artery ligation (Figure 3B). Injections were completed within 1 minute after coronary ligation. Following injection, the chest was closed, and animals were allowed to recover under a heating pad. For the PDGF-BB retention study (120 surviving rats), animals were sacrificed after 10 minutes, 1 day, 3 days, or 14 days. 
For the functional and histological studies (126 surviving rats), rats were euthanized after 1, 14, or 28 days. For the PI3K/Akt blocking (16 surviving rats) and infarct size (64 surviving rats) studies, hearts were harvested after 24 hours. For the myocardial regional blood flow study (30 surviving rats), hearts were harvested for microsphere collection after 14 days. All of the procedures were performed in a blinded and randomized manner, and data were analyzed after there were at least 6 animals in each coded group. The overall surgical mortality rate in this study was 5.3\% (20 of 376 rats, with 356 surviving rats for the study cohorts).

ELISA. For the in vivo PDGF-BB delivery study, myocardial protein was extracted from injected sites using a nonreducing buffer containing $1 \%$ Triton X-100, $50 \mathrm{mM}$ Tris (pH 7.4), $300 \mathrm{mM} \mathrm{NaCl}, 5 \mathrm{mM}$ EDTA, and $0.02 \%$ $\mathrm{NaN}_{3}$ supplemented with proteinase inhibitor cocktail (Sigma-Aldrich) at 1:200 dilution. The same amount of protein from each heart was loaded in triplicate for anti-human PDGF-BB ELISA following the manufacturer's instructions (R\&D Systems).

Fluorescence microscopy. Fixed myocardial sections were deparaffinized, rehydrated, and pretreated with boiling $10 \mathrm{mM}$ sodium citrate ( $\mathrm{pH}$ 7.2) for 10 minutes and $10 \mathrm{mg} / \mathrm{ml}$ proteinase $\mathrm{K}$ (Sigma-Aldrich) at room temperature for 10 minutes, followed by incubation with antibodies against phospho-PDGFR- $\beta$, cleaved caspase-3 (both from Cell Signaling Technology), Ki67 (Abcam), isolectin (Molecular Probes; Invitrogen Corp.), $\alpha$-SMA, tropomyosin, vimentin (all from Sigma-Aldrich) at $4{ }^{\circ} \mathrm{C}$ overnight, and then Alexa Fluor-conjugated secondary antibodies (Molecular Probes; Invitrogen Corp.). Sections were next incubated with anti- $\alpha$-sarcomeric actinin or tropomyosin (Sigma-Aldrich), followed with different Alexa Fluor secondary antibodies to obtain different fluorescence colors. After counterstaining with DAPI, sections were mounted and observed under fluorescence microscopy. For the vascular diameter measurement, sections were stained with isolectin or $\alpha$-SMA and photographed, and the diameter was measured using Image-Pro version 4.5 (MediaCybernetics).

Echocardiography. Echocardiographic acquisition and analysis were performed as previously described (34). Left ventricular fractional shortening was calculated as (EDD-ESD)/EDD $\times 100 \%$, where EDD is end-diastolic dimension and ESD is end-systolic dimension.

Immunohistochemistry. Formalin-fixed, paraffin-embedded sections were prepared for immunohistochemistry as previously described (29, 35 ). The first antibodies used were anti-phospho-PDGFR- $\beta$, anti-phospho-Akt, anti-cleaved caspase-3 (all from Cell Signaling Technology), anti-neutrophil (Serotec), anti-mac3 (BD Biosciences), and anti-BrdU (Roche Diagnostics Corp.).

Myocardial I/R and measurement of infarct size. Myocardial I/R and measurement of infarct size, as described previously (35), were performed in rats. The ischemia time was 60 minutes and reperfusion period 24 hours. All of the procedures were performed in a blinded and randomized manner, and there were at least 6 animals in each group.

BrdU protocol. Two hours before sacrifice, $50 \mathrm{mg} / \mathrm{kg}$ body weight of BrdU (Sigma-Aldrich) was injected i.p. in rats. BrdU staining was performed using the method described by Geary et al. (36). A piece of small gut from each animal was used for positive control.

DNA synthesis. Cell proliferation was measured using $\left[{ }^{3} \mathrm{H}\right]$ thymidine incorporation into DNA as previously described (37).
Myocardial regional blood flow measurement. Myocardial regional blood flow was measured using fluorescent microspheres as described by Van Oosterhout et al. (38) with modifications. Briefly, animals received heparin ( $\sim 2 \mathrm{U} / \mathrm{g}$ body weight) and were anesthetized. The entire heart was exposed via left thoracotomy, and the descending aorta was opened to insert a withdrawal catheter connecting to a bidirectional rolling pump. Blood was withdrawn at a rate of $1-2 \mathrm{ml} / \mathrm{min}$ for 2 minutes, starting 5 seconds before injection of fluorescent microspheres into the left atrium. The blood was transferred to a collecting tube containing $2 \mathrm{mg}$ of EDTA, and the total amount was recorded. A total $30 \mu \mathrm{l}$ of microspheres, a mixture of equal amount of blue-green (10- $\mu \mathrm{m}$ diameter; $3.6 \times 10^{4}$ beads), green $(15-\mu \mathrm{m}$ diameter; $1 \times 10^{4}$ beads), and yellow-green $\left(10-\mu \mathrm{m}\right.$ diameter; $\left.3.6 \times 10^{4}\right)$ beads (all from Molecular Probes; Invitrogen Corp.), was injected for each rat. Animals were sacrificed 2 minutes after injection. After removing the entire heart, the infarcted myocardium at the left ventricle was identified, harvested, weighed, and stored at $4^{\circ} \mathrm{C}$. A similar size of myocardium from the noninfarcted right ventricle was also harvested as a control. The blood and myocardium were then digested in $4 \mathrm{~N} \mathrm{KOH} \mathrm{(}>3 \times$ volume of samples) at $50^{\circ} \mathrm{C}$ for overnight. After centrifuging at $2,000 \mathrm{~g}$ for 10 minutes, the pellet that contained microspheres was washed twice with $0.25 \%$ Tween-20 and then distilled water. The pellet was dissolved with $0.5 \mathrm{ml}$ xylene and then incubated at $50^{\circ} \mathrm{C}$ for 3 hours with intermittent vortex and sonication. The supernatant was collected and subjected to measurement of fluorescence intensity (Victor ${ }^{2}$; PerkinElmer) with triplicates for each sample. The myocardial regional blood flow was calculated using the formula $Q_{\mathrm{i}}=\left(Q_{\text {ref }} \times F_{\mathrm{i}}\right) \div F_{\text {ref, }}$, where $Q_{\mathrm{i}}$ and $Q_{\text {ref }}$ are the flow rates in samples and the reference withdrawal speed, respectively, and $F_{\mathrm{i}}$ and $F_{\text {ref }}$ are the fluorescence intensity in myocardial samples and in the reference blood samples. After normalizing with weight, the absolute myocardial blood flow was expressed in milliliters/minute/gram.

Statistics. All data are expressed as mean \pm SEM. Statistical significance was determined using the 2-tailed Student's $t$ test or ANOVA as appropriate. Differences between groups were considered statistically significant at $P<0.05$.

\section{Acknowledgments}

We acknowledge the generosity of K. Walsh (Boston University School of Medicine) and T. Matsui and A. Rosenzweig (Massachusetts General Hospital, Harvard Medical School) for providing dominant-negative Akt adenovirus. P.C.H. Hsieh is supported by a postdoctoral fellowship from the American Heart Association, and M.E. Davis is a recipient of an NRSA fellowship from the National Heart, Lung, and Blood Institute. This study was supported by grants from the NIH and Medtronic.

Received for publication June 7, 2005, and accepted in revised form October 18, 2005.

Address correspondence to: Richard T. Lee, Partners Research Facility, 65 Landsdowne Street, Cambridge, Massachusetts 02139, USA. Phone: (617) 768-8282; Fax: (617) 768-8270; E-mail: rlee@ rics.bwh.harvard.edu.
1. Perin, E.C., et al. 2003. Transendocardial, autologous bone marrow cell transplantation for severe, chronic ischemic heart failure. Circulation. 107:2294-2302.

2. Kang, H.J., et al. 2004. Effects of intracoronary infusion of peripheral blood stem-cells mobilised with granulocyte-colony stimulating factor on left ventricular systolic function and restenosis after coronary stenting in myocardial infarction: the MAGIC cell randomised clinical trial. Lancet. 363:751-756. 3. Wollert, K.C., et al. 2004. Intracoronary autologous bone-marrow cell transfer after myocardial infarction: the BOOST randomised controlled clinical trial. Lancet. 364:141-148.

4. Schachinger, V., et al. 2004. Transplantation of progenitor cells and regeneration enhancement in acute myocardial infarction: final one-year results of the TOPCARE-AMI Trial. J. Am. Coll. Cardiol.
44:1690-1699.

5. Anversa, P. 2000. Myocyte death in the pathological heart. Circ. Res. 86:121-124.

6. Kang, P.M., and Izumo, S. 2003. Apoptosis in heart: basic mechanisms and implications in cardiovascular diseases. Trends Mol. Med. 9:177-182.

7. Meyer, D., and Birchmeier, C. 1995. Multiple essential functions of neuregulin in development. Nature. 378:386-390. 
8. Gassmann, M., et al. 1995. Aberrant neural and cardiac development in mice lacking the ErbB4 neuregulin receptor. Nature. 378:390-394.

9. Lee, K.F., et al. 1995. Requirement for neuregulin receptor erbB2 in neural and cardiac development. Nature. 378:394-398.

10. Gitler, A.D., et al. 2003. Nf1 has an essential role in endothelial cells. Nat. Genet. 33:75-79.

11. Chang, C.P., et al. 2004. A field of myocardial-endocardial NFAT signaling underlies heart valve morphogenesis. Cell. 118:649-663.

12. Brutsaert, D.L. 2003. Cardiac endothelial-myocardial signaling: its role in cardiac growth, contractile performance, and rhythmicity. Physiol. Rev. 83:59-115.

13. Kocher, A.A., et al. 2001. Neovascularization of ischemic myocardium by human bone-marrowderived angioblasts prevents cardiomyocyte apoptosis, reduces remodeling and improves cardiac function. Nat. Med. 7:430-436.

14. Kawamoto, A., et al. 2001. Therapeutic potential of ex vivo expanded endothelial progenitor cells for myocardial ischemia. Circulation. 103:634-637.

15. Narmoneva, D.A., Vukmirovic, R., Davis, M.E., Kamm, R.D., and Lee, R.T. 2004. Endothelial cells promote cardiac myocyte survival and spatial reorganization: implications for cardiac regeneration. Circulation. 110:962-968.

16. Kuramochi, Y., et al. 2004. Cardiac endothelial cells regulate reactive oxygen species-induced cardiomyocyte apoptosis through neuregulin-1beta/ erbB4 signaling. J. Biol. Chem. 279:51141-51147.

17. Hoch, R.V., and Soriano, P. 2003. Roles of PDGF in animal development. Development. 130:4769-4784.

18. Levéen, P., et al. 1994. Mice deficient for PDGF $\mathrm{B}$ show renal, cardiovascular, and hematological abnormalities. Genes Dev. 8:1875-1887.

19. Hellstrom, M., Kalen, M., Lindahl, P., Abramsson, A., and Betsholtz, C. 1999. Role of PDGF-B and PDGFR-beta in recruitment of vascular smooth muscle cells and pericytes during embryonic blood vessel formation in the mouse. Development. 126:3047-3055.

20. Bjarnegard, M., et al. 2004. Endothelium-specific ablation of PDGFB leads to pericyte loss and glomerular, cardiac and placental abnormalities. Development. 131:1847-1857.

21. Edelberg, J.M., et al. 1998. PDGF mediates cardiac microvascular communication. J. Clin. Invest. 102:837-843.

22. Edelberg, J.M., et al. 2002. Platelet-derived growth factor- $\mathrm{AB}$ limits the extent of myocardial infarction in a rat model: feasibility of restoring impaired angiogenic capacity in the aging heart. Circulation. 105:608-613

23. Li, X., et al. 2005. Revascularization of ischemic tissues by PDGF-CC via effects on endothelial cells and their progenitors. J. Clin. Invest. 115:118-127. doi:10.1172/JCI200519189.

24. Zhang, S. 2003. Fabrication of novel biomaterials through molecular self-assembly. Nat. Biotechnol. 21:1171-1178.

25. Ponten, A., et al. 2003. Transgenic overexpression of platelet-derived growth factor-C in the mouse heart induces cardiac fibrosis, hypertrophy, and dilated cardiomyopathy. Am. J. Pathol. 163:673-682.

26. Matsui, T., et al. 2001. Akt activation preserves cardiac function and prevents injury after transient cardiac ischemia in vivo. Circulation. 104:330-335.

27. Mangi, A.A., et al. 2003. Mesenchymal stem cells modified with Akt prevent remodeling and restore performance of infarcted hearts. Nat. Med. 9:1195-1201.

28. Bock-Marquette, I., et al. 2004. Thymosin beta4 activates integrin-linked kinase and promotes cardiac cell migration, survival and cardiac repair. Nature. 432:466-472.

29. Davis, M.E., et al. 2005. Injectable self-assembling peptide nanofibers create intramyocardial microenvironments for endothelial cells. Circulation. 111:442-450

30. Ozawa, C.R., et al. 2004. Microenvironmental
VEGF concentration, not total dose, determines a threshold between normal and aberrant angiogenesis. J. Clin. Invest. 113:516-527. doi:10.1172/ JCI200418420.

31. Xaymardan, M., et al. 2004. Senescent impairment in synergistic cytokine pathways that provide rapid cardioprotection in the rat heart. J. Exp. Med. 199:797-804.

32. Davis, M.E., Hsieh, P.C.H., Grodzinsky, A.J., and Lee, R.T. 2005. Custom design of the cardiac microenvironment with biomaterials. Circ. Res. 97:8-15.

33. Fujio, Y., and Walsh, K. 1999. Akt mediates cytoprotection of endothelial cells by vascular endothelial growth factor in an anchorage-dependent manner. J. Biol. Chem. 274:16349-16354.

34. Lindsey, M.L., et al. 2002. Selective matrix metalloproteinase inhibition reduces left ventricular remodeling but does not inhibit angiogenesis after myocardial infarction. Circulation. 105:753-758.

35. Weinberg, E.O., et al. 2005. Rosuvastatin reduces experimental left ventricular infarct size after ischemia-reperfusion injury but not total coronary occlusion. Am. J. Physiol. Heart Circ. Physiol. 288:H1802-H1809.

36. Geary, R.L., Koyama, N., Wang, T.W., Vergel, S., and Clowes, A.W. 1995. Failure of heparin to inhibit intimal hyperplasia in injured baboon arteries. The role of heparin-sensitive and -insensitive pathways in the stimulation of smooth muscle cell migration and proliferation. Circulation. 91:2972-2981.

37. Schulze, P.C., De Keulenaer, G.W., Yoshioka, J., Kassik, K.A., and Lee, R.T. 2002. Vitamin D3upregulated protein-1 (VDUP-1) regulates redoxdependent vascular smooth muscle cell proliferation through interaction with thioredoxin. Circ. Res. 91:689-695.

38. Van Oosterhout, M.F., Willigers, H.M., Reneman, R.S., and Prinzen, F.W. 1995. Fluorescent microspheres to measure organ perfusion: validation of a simplified sample processing technique. Am.J. Physiol. 269:H725-H733. 